University of Nebraska - Lincoln

DigitalCommons@University of Nebraska - Lincoln

Faculty Publications - Chemistry Department Published Research - Department of Chemistry

$4-2007$

\title{
Classification of Chemical Substances Using Particulate Representations of Matter: An Analysis of Student Thinking
}

\author{
Marilyne Stains \\ University of Nebraska-Lincoln, mstains2@unl.edu \\ Vicente Talanquer \\ University of Arizona, vicente@u.arizona.edu
}

Follow this and additional works at: https://digitalcommons.unl.edu/chemfacpub

Part of the Chemistry Commons

Stains, Marilyne and Talanquer, Vicente, "Classification of Chemical Substances Using Particulate Representations of Matter: An Analysis of Student Thinking" (2007). Faculty Publications -- Chemistry Department. 51.

https://digitalcommons.unl.edu/chemfacpub/51

This Article is brought to you for free and open access by the Published Research - Department of Chemistry at DigitalCommons@University of Nebraska - Lincoln. It has been accepted for inclusion in Faculty Publications -Chemistry Department by an authorized administrator of DigitalCommons@University of Nebraska - Lincoln. 


\title{
Classification of Chemical Substances Using Particulate Representations of Matter: An Analysis of Student Thinking
}

\author{
Marilyne Stains and Vicente Talanquer \\ Department of Chemistry, University of Arizona, Tucson, Arizona, USA \\ Corresponding author - vicente@u.arizona.edu
}

\begin{abstract}
We applied a mixed-method research design to investigate the patterns of reasoning used by novice undergraduate chemistry students to classify chemical substances as elements, compounds, or mixtures based on their particulate representations. We were interested in the identification of the representational features that students use to build a classification system, and in the characterization of the thinking processes that they follow to group substances in different classes. Students in our study used structural and chemical composition features to classify chemical substances into elements, compounds, and mixtures. Many of the students' classification errors resulted from strong mental associations between concepts (e.g., atom-element, molecule-compound) or from lack of conceptual differentiation (e.g., compound-mixture). Strong concept associations led novice students to reduce the number of relevant features used to differentiate between substances, while the inability to discriminate between two concepts (conceptual undifferentiation) led them to pay too much attention to irrelevant features during the classification tasks. Comparisons of the responses to classification tasks of students with different levels of expertise in chemistry indicate that some of these naïve patterns of reasoning may be strengthened by, rather than weakened by, training in the discipline.
\end{abstract}

\section{Introduction}

Research in science education in the past 30 years has shown that students enter science classes with many preconceived ideas about the behavior of the natural world (Wandersee, Mintzes, \& Novak, 1994; Duit, 2004). These ideas often lead learners to make predictions and build explanations different from those derived by currently accepted scientific theories and practices. Although the list of reported students' alternative conceptions in science is vast, several authors have 
suggested that many of them result from reasoning based on "common sense" (Talanquer, 2006; Chi, 2005; Driver, Guesne, \& Tiberghien, 1985; Driver, Squires, Rushworth, \& Wood-Robinson, 1997; Furió, Calatayud, Bárcenas, \& Padilla, 2000; Perkins \& Simmons, 1988; Viennot, 2001). Common-sense reasoning is grounded in a set of presuppositions about the surrounding world and the nature of things, and relies on mental strategies to make decisions and build inferences based on the available information.

Our work in recent years has been directed at identifying the common-sense patterns of reasoning used by students to analyze, predict, and explain chemical phenomena. The central goal of these studies has been to better characterize the explanatory framework of a prototypical common-sense chemistry student (Talanquer, 2006). Although we do not propose that the complexity of students' thinking in chemistry can be reduced to a limited number of basic rules or principles independent of the context or the individual, we recognize the need to develop functional models that can help chemistry teachers interpret their students' ideas in a more comprehensive way. Shifting their attention from the identification of specific alternative conceptions in a wide variety of topics to the analysis of underlying common patterns of reasoning may help chemistry teachers better identify, understand, and even predict the possible alternative conceptions that their students may hold.

In this study we continue this line of work and apply a mixed-method design approach to investigate the patterns of reasoning used by novice undergraduate chemistry students to classify chemical substances as elements, compounds, or mixtures based on their microscopic (particulate) representations. We are specifically interested in identifying the representational features that novice students use to build a classification system, characterizing the thinking processes that they follow to group substances in different classes, and comparing their performance in simple classification tasks with that of students with higher levels of preparation in chemistry. Understanding student thinking in these types of tasks is crucial since classification is one of the central aims and means of chemistry, and since a large percentage of school chemistry problems rely on the application of appropriate classification schemes.

Classification plays a central role in chemistry, where it is used not only as a way to organize knowledge, but also as a powerful predictive tool (Schummer, 1998). Chemists rely heavily on classification systems in their everyday work, from selecting a solvent in order to carry out a reaction to identifying a molecule based on a set of spectra. Unfortunately, novice learners seem to have difficulties identifying relevant categorization features when facing classification-based problems (Bransford, Brown, \& Cocking, 2000; Chi, Feltovich, \& Glaser, 1981). For example, several studies have shown that many students think of chemical compounds as mixtures of elements, and some of them classify homogeneous mixtures as pure substances (Barker, 2000; Briggs \& Holding, 1986; Sanger, 2000; Taber, 2002). However, little research has been done to uncover the origin of these difficulties and the patterns of reasoning that students follow during the classification process. 


\section{Theoretical Framework}

Research in cognitive science and science education over the past three decades has given rise to a large body of empirical and theoretical results about students' ideas of the physical world (Driver et al., 1985, 1997; Wandersee et al., 1994). Many of these studies support the view that knowledge is organized in specific domains that are characterized by a set of principles that define the entities in the domain and guide reasoning about those entities (Carey \& Spelke, 1994, 1996; Wellman \& Gelman, 1998). Individuals are assumed to be endowed with domain-specific systems of knowledge such as knowledge about physical objects and knowledge about numbers. Some of these domains are believed to be innate or developed at an early age (core domains).

Several authors have argued that knowledge in a given domain is organized in networks or explanatory frameworks that have some, but not necessarily all, of the characteristics of a theory (Gopnik \& Meltzoff, 1997; Vosniadou, 1994, 2002). These explanatory frameworks are complex systems that include perceptual information, beliefs, presuppositions, and mental representations of the entities in the domain. Although a large body of research supports the idea that prior knowledge has a strong influence on learning, researchers disagree on the degree of coherence of students' knowledge systems and on the domain-specificity of the basic presuppositions that seem to guide their thinking (Chi \& Roscoe, 2002; diSessa, 2002).

Research on human reasoning has also shown that learners make inferences about the world by using processes that are relatively simple to apply (Leighton \& Sternberg, 2004; Todd \& Gigerenzer, 2000; Tversky \& Kahneman, 1982). These shortcut reasoning procedures, also called heuristics, reduce the information-processing load. While heuristics often generate acceptable answers with little effort, they sometimes lead to severe and systematic biases and errors. Common-sense reasoning in science seems to be based on both a set of presuppositions about the physical world and the application of fast and frugal heuristics that control how and where to look for information, when to stop the search, and what to do with the results (Driver et al., 1985; Viennot, 2001). Recent work in chemical education research indicates that many of the students' alternative conceptions in chemistry may be the result of this type of reasoning (Talanquer, 2006). For example, students tend to attribute properties of material substances to abstract concepts or to processes and interactions (e.g., when students think that heat is a fluid or that chemical bonds are solid links between atoms). Students also commonly apply heuristics when analyzing causal processes. They think that the stronger, closer, or longer the duration of the cause, the larger the effect (e.g., the more electrons in an atom, the larger it is).

Common-sense reasoning is also likely applied when classifying different entities in the natural world. A large body of research in cognitive science has shown that prior knowledge strongly influences the nature and interpretation of the features that individuals select to define a class (Hayes, Foster, \& Gadd, 2003; Heit, Briggs, \& Bott, 2004; Margolis \& Laurence, 1999). The novice-expert research literature in this area indicates that novices build classification systems 
based on surface features, while expert thinking is guided by central underlying concepts (Shafto \& Coley, 2003). Research also shows that experts often create more categories than novices (Day \& Lord, 1992), and that they are able to classify just as fast at the general level as they do at the specific level (Tanaka \& Taylor, 1991). In general, experts possess a highly complex structure of knowledge organized around central ideas and concepts that help them identify more features and meaningful patterns than novices do (Bransford et al., 2000; Chi, Glaser, \& Farr, 1988). Thus, we should expect noticeable differences in the performance of novice and expert chemistry students engaged in solving chemistry problems that involve the application of a classification scheme.

\section{Methodology}

\section{Goals and Research Questions}

The central goal of this study was to investigate the thinking processes of novice undergraduate chemistry students when engaged in classification tasks involving microscopic (particulate) representations of different chemical substances (elements, compounds, and mixtures). Our investigation was guided by the following research questions:

- What are the basic features to which novice students pay attention when classifying a substance as an element, a compound, or a mixture based on its microscopic representation?

- What are the patterns of reasoning that novice students use to classify these substances into different groups or classes?

- What are the differences between the novice students' responses to the selected classification tasks and those of students with higher levels of preparation in chemistry?

\section{Context and Participants}

This study was conducted at a public Research I institution in the Southwest of the United States. The Chemistry Department at this university offers a variety of chemistry courses to over 2,500 students each semester, of whom about $50 \%$ are female and $35 \%$ are ethnically diverse students, with majors ranging from nursing and agriculture to chemical engineering.

For comparison purposes, participants in the study were drawn from six different groups representing different levels of preparation in chemistry: General Chemistry I (GCI, two sections; $n=344$ ); General Chemistry II (GCII, one section; $n=156$ ); Honours General Chemistry I (HGCI, two sections; $n=131$ ); Organic Chemistry I (OCI, one section; $n=90)$; Physical Chemistry I (PCI, one section; $n=44)$; and Graduate Students from the Chemistry Department enrolled in a College Teaching class (GS, one class; $n=39$ ). Students in the general chemistry courses (GCI and GCII) were considered as the novice chemistry students for the purposes of this study. 


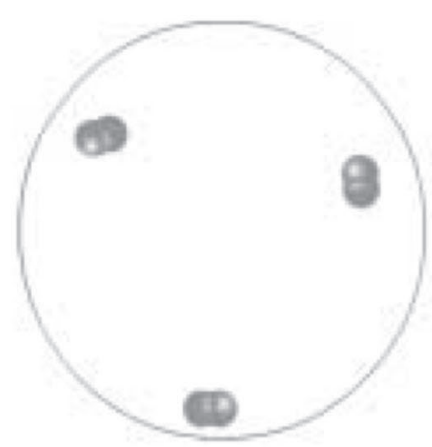

$\mathrm{E}_{2}$

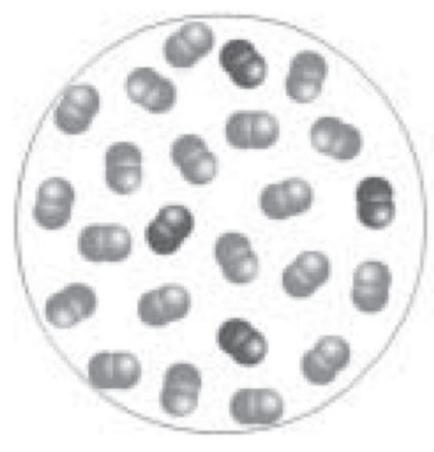

$\mathrm{C}_{2}+\mathrm{D}_{2}$

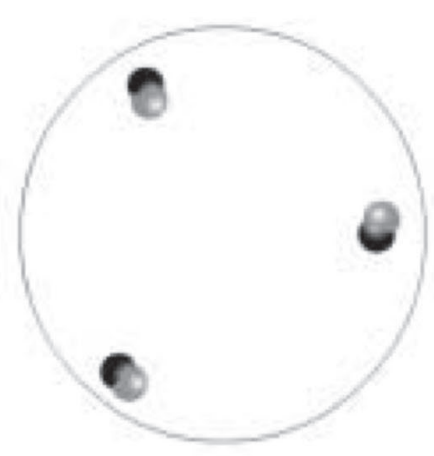

EF

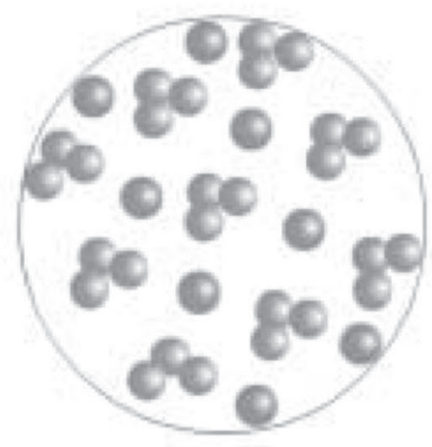

$\mathrm{C}_{3}+\mathrm{E}$

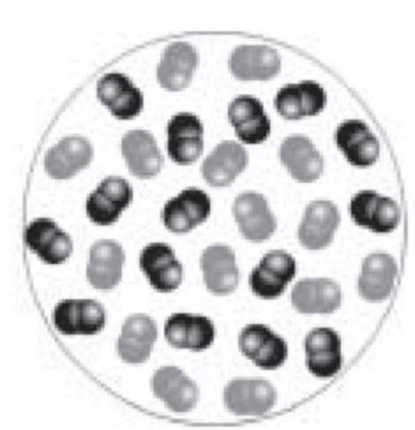

$\mathrm{E}_{2}+\mathrm{F}_{2}$

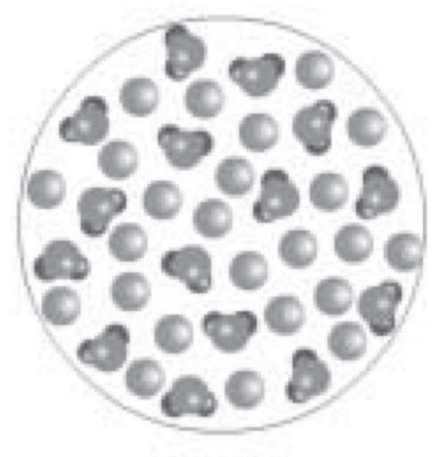

$\mathrm{D}_{2} \mathrm{E}+\mathrm{C}$

Figure 1. Examples of microscopic representations of elements, compounds, and mixtures used in our study. Each image has been labelled based on its chemical composition for reference purposes in this paper.

\section{Instruments and Procedures}

We followed a mixed-method design in which qualitative and quantitative research instruments were used (Greene, Caracelli, \& Graham, 1989). The data collection was completed in two main phases: Classification Task Questionnaires, and Interviews.

Classification Task Questionnaires. Over the course of two semesters, we visited all the chemistry sections outlined above. Students in these courses were asked to classify a set of 20 particulate images of different chemical substances as elements, compounds, or mixtures (see Figure 1 and Table 1). The images, which use circles of various color to represent atoms (or ions by adding + or - signs) of different elements, were projected one by one on a large screen for 15-s intervals. Students recorded their responses on an answer sheet that was collected immediately after the presentation of the last image. It is important to point out 
Table 1. Percentage of students who classified a given particulate representation as element, compound, or mixture in the Classification Task Questionnaire (correct answers are highlighted). Each image has been labelled based on its chemical composition for reference purposes in this paper. * Images used in the interviews

\begin{tabular}{|c|c|c|c|c|c|c|c|c|c|c|c|c|c|c|c|}
\hline & & 0다 & DEI & HDE & 6.1 & PCi & 98 & & & 대 & a대 & Hat & 0 . & $\mathrm{Mb}$ & ㅁ. \\
\hline & Emanth & 421 & 푬요 & 91 & 7 . & $16 \mathrm{~d}$ & 41 & 4 & Finat & 211 & 24 & II & EH & 45 & 51 \\
\hline & Compaund & 03 & $\pi$ & 묘 & 11 & bij & 91 & & Danpo.nd & 71 & 104 & पile & Wa & 4i] & 123 \\
\hline if & Mutim & 016 & Pi & at & 11 & Dii & 21 & EF & Minure & IT & 45 & 21 & A7 & 21 & 26 \\
\hline a & Ennuif & $\sqrt{10}$ & 71 & III & 7 7I. & $\sqrt{n i s}$ & $\sqrt{4-1}$ & Sil & Ehmit & 47 & $\pi$ & at & 13 & 21 & 26 \\
\hline & Compuni: & 107 & 247 & 215 & 219 & 245 & 636 & $4 y$ & Pancoind & 14.4 & WHA & 0.2 & 1.t. & Wi] & $44 T$ \\
\hline$E_{i}$ & Wharn & d. & 1 & at & dij & bij & of & DE & Whinis & 41 & 51 & 21 & 는 & 21 & 11 \\
\hline An & Einin: & 41 & 17 & 784 & $\pi 7$ & 704 & Mit & & 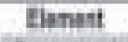 & 11 & 77 & III & 16 & i: & 100 \\
\hline & Gorpaind & al & 1E) & at: & 147 & 25 & 195 & & Gampinal & in & 164 & (11) & Wh & ait. & Hu \\
\hline Ii & Mritur & 17 & 18 & 매: & 9 E & bid & ab & $t D_{1}$ & tistuin & 23 & 11 & 15 & AT & 21 & 26 \\
\hline 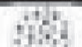 & Pinntit & 81 & 14. & 45 & If & 71 & 24 & & Tisnat & 23 & 1) & 0.0 & 11 & (1) & 10 \\
\hline 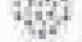 & Cortpaune & 71 & en & at & 123 & 44 & 11 & & Canpourd & 2yt & 124 & 15: & 117 & 41 & 75 \\
\hline If & Mitwe & 1 & 71 & Q17 & $12 \%$ & FI & $B 1$ & IE & Min'in: & iil! & 141 & Ai1 & Hi & Bil & 71 \\
\hline & Einniti & 61 & II & 21 & 11 & Di4 & 00 & & Ehminal & 70 & 37 & 40 & 1.1 & 9! & 76 \\
\hline$x$ & Gompuns & 15 & 111 & 31 & 44 & 23 & $M$ & & Auncond & +40 & 16 & 125 & E7 & 4 & 40 \\
\hline DiE & Mintur & 73 & 83 & 54 & 14 & $\mathrm{Di}$ & 43 & EFit & Nituin & Hil & 14 & 611 & 13 & $\sqrt{15}$ & 114 \\
\hline ith & Ein & A1 & 111 & 21 & 11 & bit: & 24 & it & Finnit & 15 & $2 H$ & DIH & P16 & 0 is & 26 \\
\hline wit & Compeine & 96 & 17 & d? & 111 & $j$ & id: & & Campoind & III & it? & 6.) & 73 & 11 & 76 \\
\hline Eiph & Mithrie & 115 & 111 & all & Ir: & त? & ait & $\mathrm{C}+\mathrm{EF}$ & Nictun & 111 & 117 & 918 & ail & प17 & 배물 \\
\hline Hin & Euminl & 16 & III & 01 & 21 & bit & 06 & & Emant & 4J & 43 & ib & it & it: & 40 \\
\hline & Crorpaune & 140 & 71 & 61 & 156 & 71 & 31 & & Cenponad & 17 & 11 & Si & 711 & 197 & 70 \\
\hline$E_{i}+F_{i}$ & Matere & 08 & 411 & 412 & 123 & 177 & M4 & DHEE & Whin & 126 & BI & Tif & 7 thit & B.1 & 172 \\
\hline$A 4$ & Barint & 72 & II & it: & 12 & Did & 015 & & Entuat & II & Did & (1) & 11 & it: & 10 \\
\hline 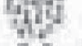 & Compun! & $\mathrm{It}$ & 15 & $4 !$ & 122 & 21 & 51 & & Cupporal & 118 & 57 & 36 & 11.1 & 61 & 26 \\
\hline $\mathrm{Er} \mathrm{D}_{4}$ & Wilum & at & II: & BS & AIf & Wit & H & $A=A$ & Histun & 415 & this & (1) & DA & 81 & 114 \\
\hline PE & Einint & 21. & 21 & DI & 11 & Bid & 01 & & Ehmit & 17 & 18 & 00 & 11 & 0. & 90 \\
\hline if & Curpaint & tis & DI & it & $H 1$ & BS & 316 & 47 & Canpuind & ind & 43i & 30 & 44 & H. & 2.5 \\
\hline Endt & Witur & $\pi$ & 42 & 191 & 110 & 142 & 19: & Eif & Wirtuin & 81 & 44 & FD & 111 & W1 & ins \\
\hline & Dinent & 70 & 1.8 & 10. & $7 B$ & fis & 24 & & Thmat & 0.5 & [1] & 106 & 12 & 0. & 10 \\
\hline & Empquil: & nt & 74 & iㅐ연 & 144 & HI & HI & ings & Bampoird & 211 & 154 & 11 & 11 & il & 21 \\
\hline Hins: & Matart & 67 & 15 & 4 & 71 & Dill & 21 & EFDE & Hintur & 7.1 & 14it & 917 & Fil & mI & 71 \\
\hline
\end{tabular}

that the distinction between elements, compounds, and mixtures is a topic traditionally addressed within the first three weeks of the GCI course. Our study was conducted in the second half of the academic semester, and thus students in this group (GCI) had already been exposed to this topic during the regular lecture.

Interviews. Twenty-eight volunteers from the General Chemistry classes (19 students from GCI and nine from GCII) involved in the first phase of the study completed a 1-h semi-structured interview. Each interview was tape-recorded and transcribed. Three slightly different interview protocols were used to investigate several aspects of students' thinking while also limiting the cognitive de- 
mand on each interviewee. Students were randomly assigned to one of the protocols. All protocols included a common set of activities that have proven to be effective in eliciting student thinking and cognitive structure (Tsai \& Huang, 2000; White \& Gunstone, 1992), such as the construction of concept maps (Novak, 1990) and pictorial representations (Symington, Boundy, Radford, \& Walton, 1981). The main difference between protocols was based on the completion of an additional exploratory activity, such as a word association task or a word definition task (Preece, 1976).

As part of all of the interviews, the 28 participants were asked to draw microscopic representations of an element, a compound, and a mixture, and to develop a concept map based on these concepts. All interviewees were also asked to classify 15 of the 20 microscopic representations used in the first phase of the project (we selected the 15 microscopic representations that had been misclassified most frequently in the classification task questionnaires described earlier). Students were given the 15 images of different chemical substances and asked to classify them in different groups, thinking out-loud during the process. In Protocols A and C, students' answers were constrained by specifying that they should classify the microscopic representations as elements, compounds, or mixtures; while in Protocol B, students self-defined their groups. This latter variation in the protocol allowed us to investigate to what extent participants spontaneously used elements, compounds, and mixtures as organizing classes.

In two of the interview protocols (Protocols A and B; 19 students), students were additionally asked to define the terms element, compound, and mixture in their own words, while in the third protocol (Protocol C; nine students) they had to write the first word that came to their mind when they heard each of these concepts (word association task). The answers to these additional tasks were mainly used to validate conclusions derived from the analysis of results from those core tasks that were common to all of the protocols. For reference and privacy purposes, a code was created to label each of the participants; this code has been used throughout the discussion of our results. The assigned label is based on the order of the interview and the type of protocol used. For example, Interviewee S1A refers to the first (1) subject (S) who completed the interview Protocol A.

\section{Data Analysis}

Grounded theory (Charmaz, 2003) was used to analyze the interview transcripts and other forms of evidence. In this iterative, non-linear analysis, categories were generated by highlighting common statements and major ideas relevant to the research questions. Each activity included in the interview protocols revealed their own set of categories, which then were regrouped according to common themes. Main categories were derived from the analysis of results for activities common to all protocols; results for other tasks were mainly used for validation purposes. This process together with the statistical analysis of the answers to the classification task questionnaire allowed us to identify both the central features that students used to build their classification schemes as well as the main patterns of reasoning guiding their decisions. 


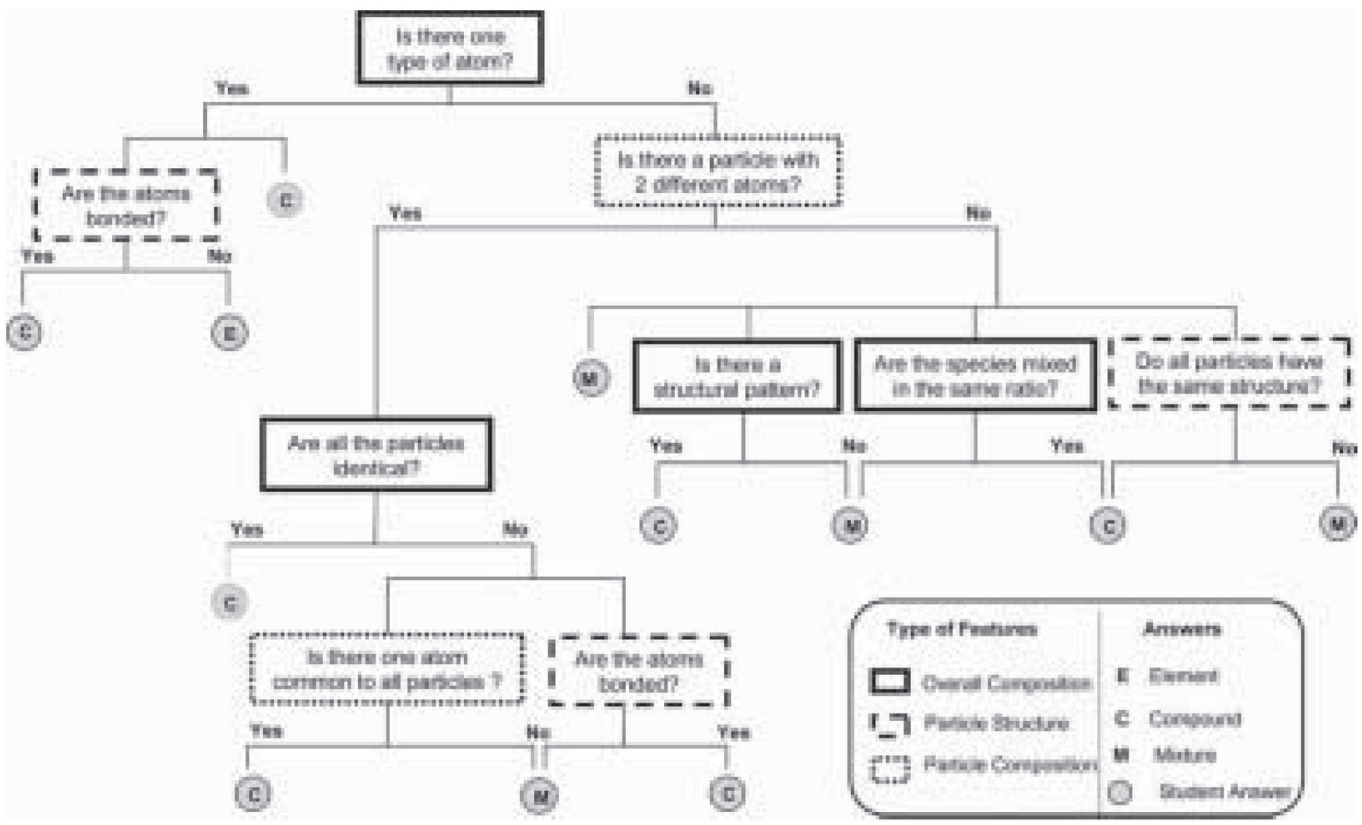

Figure 2. The diagram summarizes the types of features used by students to classify microscopic representations of chemical substances into elements (E), compounds (C), and mixtures (M).

Additional validation and extension studies involving another group of 32 GCII students and five "expert" chemists (chemistry faculty and instructors) were conducted in the final stages of the data analysis to test the validity of our major inferences and claims. Students were asked to classify as element, compound, or mixture 15 microscopic representations specifically designed to test the basic results of our analysis. The five expert chemists completed a 30-min interview using the set of activities included in interview Protocol B (which is based on selfdefined groups for the main classification task).

\section{Results and Discussion}

\section{Features}

Figure 2 compiles the representational features that the interviewed students took into consideration when classifying chemical substances into elements, compounds, or mixtures based on their microscopic representations. We have used a question format to highlight the types of features to which students paid attention when making judgements and decisions during the classification tasks. The analysis of the interview transcripts and the students' concept maps and drawings shows that most students based their classification on three main types of 
features: the overall structure and composition of the microscopic representation (overall composition), the atomic composition of each particle in the system (particle composition), and the presence of bonding between atoms (particle structure). Figure 2 depicts the specific features that students considered during the activities associated with the different interview protocols. However, not all of the students based their decisions on the analysis of each feature in this diagram.

\section{Patterns of Reasoning}

In general, misclassification of a substance commonly occurred when students reduced the number of relevant classification features or failed to differentiate between the concepts of element, compound, and mixture. Thus, we selected the ideas of reduction and undifferentiation as the basis for the categories used to discuss the main results of this part of the study.

\section{Reduction}

The tendency of students to reduce the number of variables considered when making a decision is characteristic of common-sense learners (Driver et al., 1985; Viennot, 2001). This pattern of reasoning was mainly observed in the classification of elements and compounds. In general, reduction was based on the search for a single distinctive feature that was strongly associated with each type of substance: identical isolated atoms in the case of elements, and bonded particles (molecules) in the case of compounds. For example, during the interviews, more than one-quarter of the students (5 out of 19) who were asked to define an element clearly thought that they were composed of single atoms and then used this as the unique criterion in differentiating elements from other substances. Moreover, 18 out of 28 interviewed students chose to draw a single circle to represent a chemical element, and four of the nine students who completed the word association activity wrote "single" or "single atom" after hearing the word element.

The association between the concepts of atom and element was also evident in 10 of the 28 concept maps created by the participants in our study, as illustrated by the conceptual links shown in Figure 3. Several students thought of elements as the common label used to refer to the individual atoms represented in the periodic table. Indeed, 10 of the 28 students related the word element to the words periodic table in their concept maps and 5 of the 19 definitions of the concept element referred to the "periodic table." Although these relationships are not necessarily incorrect, they reflect the students' strong association between the concepts atom and element.

Additionally, almost one-half of the students who were asked to define the concept of compound during the interviews thought of these substances as something that "has bonds;" more than one-third of them defined mixtures as something that "is not bonded or not interacting." The presence of bonded particles or molecules as the distinctive feature of chemical compounds surfaced repeatedly in the different activities associated with the three interview protocols. Sev- 

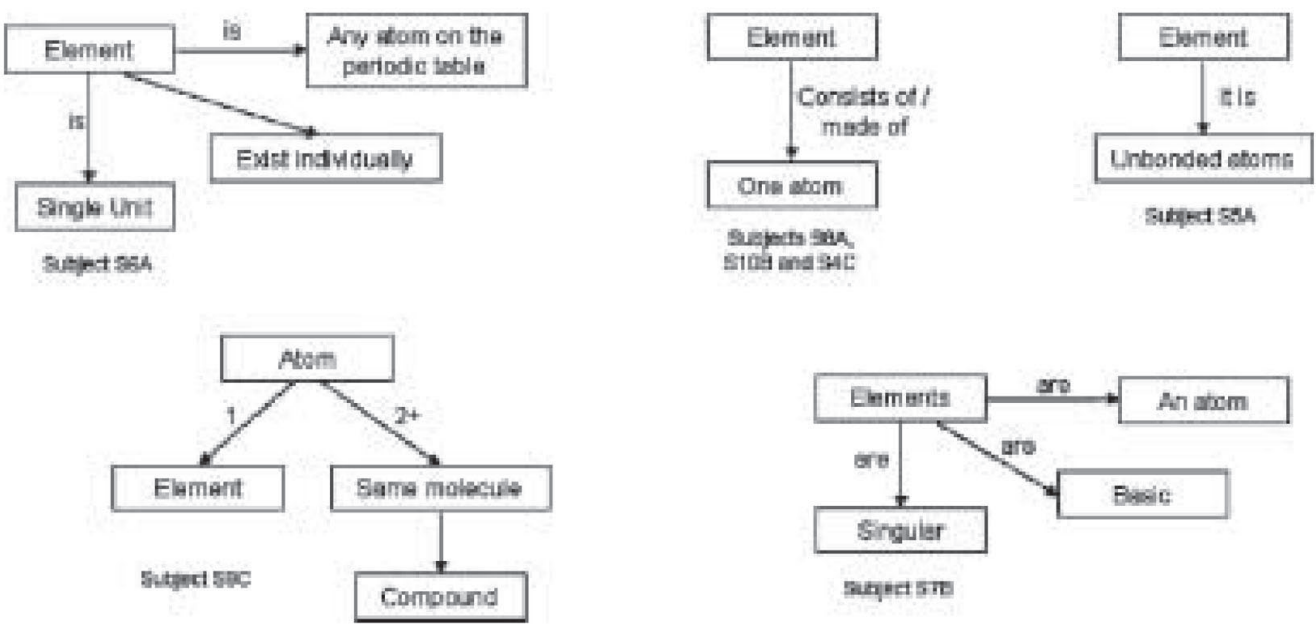

Figure 3. Excerpts from seven concept maps that illustrate the association between the concepts atom and element

enteen of the 28 students drew a single molecule with two or more different atoms to represent a compound; only six of the students drew an ionic compound. During the word association activity, four out of nine students wrote "water" after hearing the word compound, and nine of the 28 students linked the concepts compound and molecule in their concept maps. One of the interviewed students clearly described the difficulty he had in overcoming the strong association between the concepts molecule and compound in the classification of molecular elements such as E2:

People have to tell me over and over that elements exist bonded like $\mathrm{O}_{2} \ldots$ I would more easily put it in compound because of the bonding. So, when I am on a test, I need to think hard that a diatomic molecule, with the same atoms, is an element. (S7A)

The strong mental association between the concepts of compound and molecule could be used to explain why a significant proportion of students misclassified molecular elements $\left(\mathrm{E}_{2}\right.$ and $\mathrm{F}_{4}$ in Table 1$)$ as chemical compounds in the classification task questionnaire. Surprisingly, this association seemed to be stronger among students with more advanced training in the discipline, as illustrated in Figure 4 where we show the percentage of students in different groups who classified the species $E_{2}$ as an element or a compound. Analysis of the data using a chi-square test reveals that there is a statistically significant relationship $(p<0.0001)$ between the number of students who classify $\mathrm{E}_{2}$ or $\mathrm{F}_{4}$ as an element or as a compound and the level of chemistry expertise. To identify which groups were the major contributors to the statistically significant difference in the data, we performed a post hoc test to evaluate the standardized residuals for each group (Lawal, 2003). This analysis showed that students in General Chemistry (GCI), students in more advanced chemistry courses (GCII, HGCI, OCI, PCI), and graduate students in chemistry (GS) differed significantly in their responses to the classification task. 


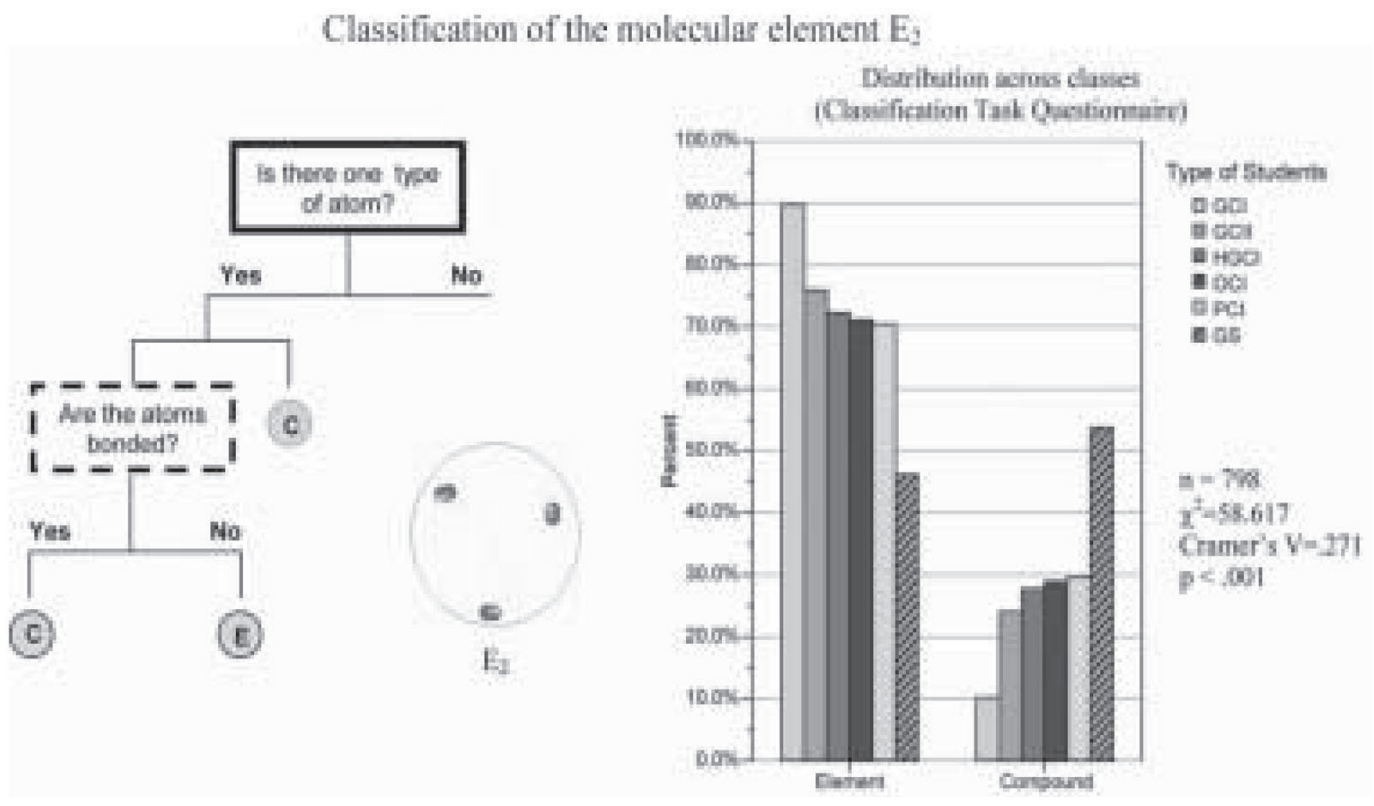

Figure 4. Percentage of students with different levels of preparation who classified the molecular element $E_{2}$ as element, compound, or mixture in the Classification Task Questionnaire (see Table 1). The diagram on the left illustrates the types of features that may have influenced the students' decisions (as suggested by student interviews). Relevant information from the statistical analysis of the data is included

The observed significant difference in the responses of novice students in GCI and in more advanced chemistry courses (GCII, HGCI, OCI, PCI) may be the result of shallow learning in the introductory course. The topic of elements, compounds, and mixtures is the subject of direct instruction in GCI, and this may explain the larger fraction of students in this group who correctly classify $E_{2}$ and $F_{4}$ as elements. However, if learning for some of these students is based on rote memorization rather than on deep understanding of basic chemical concepts, it is likely they will revert to their prior conceptions or knowledge later on.

There is also a significant difference in the results for graduate students and undergraduate students in advanced chemistry classes. Although graduate students' responses to the classification task questionnaire could also be an indication of shallow learning in their introductory chemistry courses, our study points to other possible causes. Short informal conversations with graduate students revealed that some of them thought of the term element as the common label for the different types of atoms represented in the periodic table, rather than as a type of chemical substance.

For many of them, the particulate representation of a substance seemed to trigger an alternative classification system based on molecular structure rather 
than on chemical composition. We observed a similar phenomenon in the interviews with expert chemistry faculty. During the construction of their own classification system, most experts based the categorization of pure substances on molecular structure rather than chemical composition. For example, one of the interviewees grouped $\mathrm{E}_{2}$ and $\mathrm{EF}$ together as "diatomic" particles, and some of the participants went so far as to associate the label compound with any non-monoatomic entities. The following quotes illustrate the types of ideas expressed by three out of five chemistry faculty during the classification task:

...if you think of elements in the context of real materials like sulphur $\mathrm{S}_{8}$ then it's different... we call sulphur an element but it's really a compound... an element is one atom but a compound is more than one. (F1)

An element is just one single species. I think of something that shows up in the periodic table; a single atom. An element cannot be diatomic; it would be a compound. (F2)

These types of results suggest that the unexpected increase in the percentage of "experts" who classify $\mathrm{E}_{2}$ as a compound is not the result of a simple reversion to prior ideas, but rather is more closely associated with a shift of focus from composition to structural features in the identification of relevant classification factors. From this new perspective, the classification of $E_{2}$ as an element is seen by experts as having little use or as being irrelevant. In analyzing systems at the particulate level, the structure and complexity of the particles becomes of central interest, and substances such as $\mathrm{E}_{2}$ and $\mathrm{EF}$ have more in common than $\mathrm{E}_{2}$ and $\mathrm{E}$. In this structure-based framework, the word compound is redefined and used in the same way as many naïve learners do: to refer to substances comprised of identical molecules, regardless of their composition. The concept of element is then reserved to refer to the different types of atoms that compose a molecule.

\section{Undifferentiation}

A variety of studies show that common-sense learners do not clearly differentiate between certain concepts, such as heat and temperature or speed and velocity, and tend to apply fused or coalesced notions to interpret or explain physical phenomena (Smith, Carey, \& Wiser, 1985). In our study, this type of conceptual undifferentiation was demonstrated in various instances by several of the interviewed students who did not clearly differentiate between the concepts compound and mixture. For example, two of the nine students who completed the word association activity connected the word compound to the word mixture, and two other students linked the word mixture to the chemical formulas $\mathrm{HCl}$ and $\mathrm{NaCl}$. Six of the 28 interviewed students drew either an actual compound to represent a mixture or, vice versa, a mixture as a representation of a compound. During the interviews, 17 of the 28 students made comments that revealed some level of undifferentiation between the concepts compound and mixture. The following excerpts from the student interviews illustrate this confusion: 
$\mathrm{HCl}$ is a mixture too I guess... a mixture is a compound... I think they are synonymous with each other or something like that... like if I have a container full of carbon dioxide it's a mixture because I hold it into my hand but when I write it down then it's a compound. (S7B)

A compound is a mixture of two different elements. (S8B)

A compound is just two elements mixed together... I think that compounds are mixtures. (S3A)

I can't make a difference between compound and mixture; I still don't know what a mixture is. I can't think of it at the microscopic level... Water is a compound but it could be kind of a mixture too. Compound and mixture are the same to me. (S2A)

The analysis of the students' verbalized thinking during the interviews suggests that the undifferentiation between the concepts compound and mixture led many students to consider extraneous features during the classification of these types of substances. In particular, we identified two main distracting factors: the presence of common atoms among different particles, and the relative concentration and distribution of the particles in the system. We analyze the corresponding evidence in the following paragraphs.

Common atoms. The analysis of the students' responses to the classification task administered during the first phase of our study revealed that mixtures of two types of particles in which one of the species is part of the other $(\mathrm{DE}+\mathrm{E}, \mathrm{D}+$ $\mathrm{D}_{2} \mathrm{E}$, and $\mathrm{EF}+\mathrm{E}_{2} \mathrm{~F}$ in Table 1 and Figure 1) were classified as compounds by up to $40 \%$ of the students in general chemistry. Our results are summarized in Figure 5, where we can see that although the percentage of students who misclassified these particulate representations as compounds decreases in a significant manner $(p<0.001)$ with further training in the discipline, the actual percentage value remained relatively high across the different groups.

Individual interviews revealed that some students thought of these types of mixtures as "reacting systems" where the smaller particles are either left over from an incomplete reaction or have the potential to form more product (the other substance). The classification of these types of representations as chemical compounds was based not on the actual composition of the system, but rather on the assumption that a reaction had occurred and the only relevant substance was the compound identified as the product, and the perceived potentiality of the system to become a compound if more reactants were added to the system. The following excerpts illustrate these ideas:

All the blues are bonded to the red and there is a bunch of red left over but if there were more blues they would bond... you have one type of element left over so it could kind of look like a mixture because there are not all bonded but because you have just one type of elements left over and not others then it will be a compound. (S8A's explanation during the classification of the system DE + E)

You have the two different elements bonding and there is blue left over but there is no free red for them to bond to... the extras don't really count. (S8A's explanation during the classification of $\mathrm{D}+\mathrm{D}_{2} \mathrm{E}$ ) 


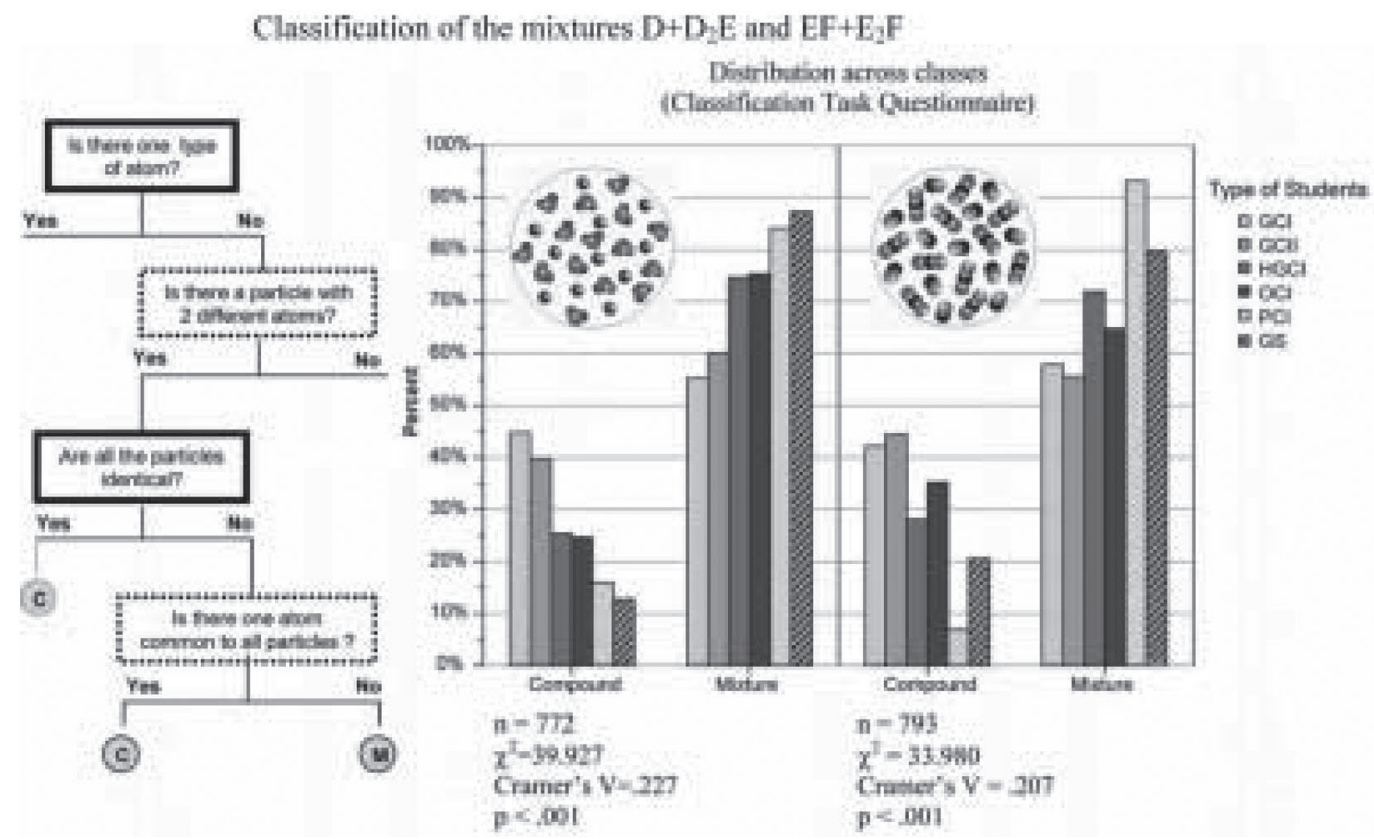

Figure 5. Percentage of students with different levels of preparation who classified the systems $\mathrm{D}+\mathrm{D}_{2} \mathrm{E}$ and $\mathrm{EF}+\mathrm{E}_{2} \mathrm{~F}$ as element, compound, or mixture in the Classification Task Questionnaire (see Table 1). The different particles in these systems share common atoms. The diagram on the left illustrates the types of features that may have influenced the students' decisions (as suggested by student interviews). Relevant information from the statistical analysis of the data is included.

Particle concentration and distribution. Several students classified as compounds the particulate representations of homogeneous mixtures of elements present in the same ratio $\left(\mathrm{C}+\mathrm{F}, \mathrm{E}_{2}+\mathrm{F}_{2}\right.$ in Figure 1 and Table 1$)$ or those of mixtures of particles arranged in regular patterns $\left(\mathrm{C}_{3}+\mathrm{E}\right)$. Although the fraction of students who misclassified mixtures as compounds based on the relative concentration or the distribution of particles in the system was less than $20 \%$ for most groups (see Table 1), individual interviews revealed that a significant proportion of the students seemed to pay attention to these features during the classification process. For example, 10 of the interviewed students referred to the concentration or distribution of particles as features that they considered in the classification of the particulate representations of chemical substances. The idea that "a compound always contains the same ratio of its component atoms" was misinterpreted by students who thought that all systems with particles present in a one to one ratio were chemical compounds (in particular, ionic compounds): 
I thought that it may be a compound because there was the same number... so it may be ionic... If there is a one to two ratio or something like that, I would probably put it with the compound. I'm not too confident about $1 \mathrm{~B}, 1 \mathrm{~F}$ and $2 \mathrm{E}$ [referring to systems $\mathrm{C}+\mathrm{F}, \mathrm{E}+\mathrm{D}_{2}$, and $\mathrm{E}_{2}+\mathrm{F}_{2}$ ] $\ldots$ there is the same number so it could be ionic; the ratio makes me doubt. (S6C)

The influence of this idea can be confirmed by comparing students' responses to the classification of mixtures $E_{2}+F_{2}\left(1: 1\right.$ particle ratio) and $C_{2}+D_{2}$ (5:1 particle ratio) included in the classification task questionnaire. For all groups, the percentage of students who misclassified the first mixture as a compound was systematically higher (up to $4.5 \%$ for students in GCI and GCII and students in the OCI) than those who made the same mistake with the latter mixture (see Table 1). For novice students in GCI, there is a significant difference $(p<0.1)$ between the number of students who misclassified the 1:1 mixture $E_{2}+F_{2}$ as a compound or as an element and those that misclassified the 5:1 mixture $C_{2}+D_{2}$ in a similar way. The presence of equal number of particles of two different substances seemed to mislead novice students, who classified the mixture as a chemical compound.

The spatial distribution of particles is a feature that also seemed to influence the participants' classification decisions. More than one-half of the interviewees who classified the system $\mathrm{C}_{3}+\mathrm{E}$ as a compound (see Figure 1) indicated that the structural pattern was characteristic of ionic compounds. The following interview excerpt illustrates this idea:

I look for structure if there is a structure then it obviously... could be an ionic compound; if there is no pattern then it's a mixture. (S9C)

Some of the students who associated the presence of a structural pattern with ionic compounds implicitly assumed that the represented atoms or particles had a positive or negative charge, although these charges were not included in the particulate representations. These novice students tended to neglect or misinterpret important representational features, as evidenced in the following excerpts from the interviews:

It might be an ionic compound because if these (red) are positive and these (green) are negatives then they are forming a certain shape, they have a pattern... but I don't know... it could be an element with another element but then this could also be an ionic compound. (S4A)

The pattern could be like dispersion forces or like mild attraction of the electron cloud so it could be a compound, but I guess it's not well... I don't know... I'm not sure on that one because it has the two different elements and they are not bonded at all cause they are not overlapping which make me think it was a mixture but there is a definite pattern to it, so I don't know if it's just how it's suppose to be or if there is some sort of attraction that made it going to that pattern. (S8A)

\section{Conclusions}

Novice students in our study used structural and chemical composition features to classify chemical substances into elements, compounds, and mixtures 
based on particulate representations. However, for many of them the microscopic representations of these systems seemed to trigger a classification scheme in which structural features, such as the bonding between particles, the distribution of particles in space, or the presence of common atoms among different species, were more relevant than characteristics associated with chemical composition.

Many of the students' classification errors occurred when students either reduced their decision to the identification of a single feature that was used to define a class (e.g., assuming that all systems with bonded particles are compounds) or did not clearly differentiate between concepts (e.g., assuming that compounds are like mixtures). The lack of differentiation between concepts was many times associated with the misinterpretation of formal definitions (e.g., "a compound always contains the same ratio of its component atoms") or the overgeneralization of ideas (e.g., "particles in solid ionic compounds are arranged in regular patterns"). Misinterpretation and overgeneralization led many students in our study to consider a variety of irrelevant features in making their classification decisions (e.g., presence of common atoms among different particles, distribution of particles in the system, relative concentration of different species).

Our results indicate that a significant proportion of students had developed strong associations between the concepts atom and element, and molecule and compound, that influenced their classification decisions. Surprisingly, these mental associations seemed to be strengthened rather than weakened by further training in the discipline. However, it is very likely that the association established by more advanced students and even the expert chemistry faculty is based on the application of a knowledge framework in which structural features become central to the categorization of chemical substances at the particulate level. The concept of compound, in opposition to that of mixture, was more frequently associated with terms such as homogenous, equal ratio, structure, pattern, and bonded. Our results also showed that students were also more likely to classify a substance as a chemical compound when there was a similarity among particles; in particular, in cases where one type of particle resembled a part of another type of particle also present in the system. In this case, classification judgements seemed to be based on an erroneous interpretation of the system as an incomplete chemical reaction that had the potential to produce a single compound.

In general, our results reinforce the conclusions from previous studies on the differences between novice and expert thinking (Bransford et al., 2000; Chi et al., 1988). Novice chemistry students in our study lacked an integrated structure of knowledge about chemical substances that allowed them to identify meaningful patterns and to differentiate relevant from extraneous classification features. Their mental organization of knowledge was rather crude and diffuse, based on strong but sometimes false concept associations and highly undifferentiated concepts (Smith et al., 1985).

In order to test further the validity of our central claims concerning the extraneous factors that influenced students' classification of compounds and mixtures, we conducted a small extension study involving a group of 32 students enrolled in GCII during a subsequent semester. In this case, students were asked to classify as elements, compounds, and mixtures a group of particulate representations 
Table 2. Percentage of students who classified the different particulate Representations A, $\mathrm{B}$, and $\mathrm{C}$ as elements, compounds, or mixtures in the extension study

\begin{tabular}{l|ccc}
\hline & & & \\
& $\mathrm{A}$ & $\mathrm{B}$ & $\mathrm{C}$ \\
\hline Element & $3.1 \%$ & $3.1 \%$ & $0.0 \%$ \\
Compound & $3.1 \%$ & $\mathbf{1 5 . 6} \%$ & $56.2 \%$ \\
Mixture & $93.8 \%$ & $81.3 \%$ & $43.8 \%$ \\
\hline
\end{tabular}

specifically selected based on the analysis and conclusions summarized in previous paragraphs. Table 2 summarizes the relevant results of this extension study.

The results depicted in Table 2 follow a trend that is consistent with the main conclusions of this paper. We expected more students to classify particulate Representation B as a compound than Representation A because Representation B is composed of "bonded" particles with identical structure present in "equal ratios". Although particle Representations B and C share similar features, Representation $C$ is more misleading because it includes one type of particle that looks like a piece of the other type. Thus, we anticipated that some students would probably think that the smaller pieces had the "potential" to combine to form the larger particles. As shown in Table 2, more than one-half of the respondents erroneously classified this substance as a compound.

The analysis of student thinking during the classification tasks associated with this study revealed common patterns of reasoning that should be taken into account in the design of instructional activities to promote meaningful learning about the distinctive features of chemical substances at the microscopic level. Students may benefit from instruction that challenges some of the strong mental associations identified in our study, and that makes them compare and contrast the distinctive features of elements, compounds, and mixtures at the microscopic level. The successful use of classification schemes to solve problems in chemistry depends on the students' ability to clearly identify relevant features that differentiate groups or classes. Our study suggests that common-sense learners will tend to reduce the complexity of the task by using a single classification criterion, or will pay too much attention to extraneous features due to a lack of clear differentiation between concepts. However, more research is needed to better characterize student thinking when solving classification-based chemistry problems.

\section{References}

Barker, V. (2000). Beyond appearances: Students' misconceptions about basic chemical ideas. Retrieved August 25, 2005, from http://www.chemsoc.org/pdf/LearnNet/rsc/ miscon.pdf 
Bransford, J. D., Brown, A. L., \& Cocking, R. R. (editors). (2000). How People Learn: Brain, Mind, Experience, and School. Washington, DC: National Academy Press.

Briggs, H., \& Holding, B. (1986). Aspects of Secondary Students' Understanding of Elementary Ideas in Chemistry: Full Report (Children's learning in science project). Leeds: University of Leeds.

Carey, S., \& Spelke, E. (1994). Domain-specific knowledge and conceptual change. In: L. Hirschfeld \& S. A. Gelman (editors), Mapping the Mind. New York: University of Cambridge.

Carey, S., \& Spelke, E. (1996). Science and core knowledge. Philosophy of Science, 63, 515-533.

Charmaz, K. (2003). Chapter 8: Grounded theory-Objectivist and constructivist methods. In: N. K. Denzin \& Y. S. Lincoln (editors), Strategies of Qualitative Inquiry. Thousand Oaks, CA: Sage.

Chi, M. T. H. (2005). Commonsense conceptions of emergent processes: Why some misconceptions are robust. Journal of the Learning Sciences, 14(2), 161-199.

Chi, M. T. H., Feltovich, P. J., \& Glaser, R. (1981). Categorization and representation of physics problems by experts and novices. Cognitive Science, 5(2), 121-152.

Chi, M. T. H., Glaser, R., \& Farr, M. J. (editors). (1988). The Nature of Expertise. Hillsdale, NJ: Erlbaum.

Chi, M. T. H., \& Roscoe, R. D. (2002). The process and challenges of conceptual change. In: M. Limón \& L. Mason (editors), Reconsidering Conceptual Change: Issues in Theory and Practice. Dordrecht, The Netherlands: Kluwer Academic.

Day, D. V., \& Lord, R. G. (1992). Expertise and problem categorization-The role of expert processing in organizational sense-making. Journal of Management Studies, 29(1), 35-47.

diSessa, A. (2002). Why "conceptual change" is a good idea. In: M. Limón \& L. Mason (editors), Reconsidering Conceptual Change: Issues in Theory and Practice. Dordrecht, The Netherlands: Kluwer Academic.

Driver, R., Guesne, E., \& Tiberghien, A. (1985). Children's Ideas in Science. Milton Keynes, England: Open University Press.

Driver, R., Squires, A., Rushworth, P., \& Wood-Robinson, V. (1997). Making Sense of Secondary Science: Research into Children's Ideas. London: Routledge.

Duit, R. (2004). Bibliography STCSE: Students' and teacher's conceptions and science education. Retrieved August 25, 2005, from http://www.ipn.uni-kiel.de/aktuell/stcse/

Furió, C., Calatayud, M. L., Bárcenas, S. L., \& Padilla, O. M. (2000). Functional fixedness and functional reduction as common-sense reasonings in chemical equilibrium and in geometry and polarity of molecules. Science Education, 84(5), 545-565.

Gopnik, A., \& Meltzoff, A. N. (1997). Words, Thoughts, and Theories. Cambridge, MA: MIT Press.

Greene, J. C., Caracelli, V. J., \& Graham, W. F. (1989). Toward a conceptual framework for mixed-method evaluation designs. Educational Evaluation and Policy Analysis, 11, 255-274.

Hayes, B. K., Foster, K., \& Gadd, N. (2003). Prior knowledge and subtyping effects in children's category learning. Cognition, 88(2), 171-199.

Heit, E., Briggs, J., \& Bott, L. (2004). Modeling the effects of prior knowledge on learning incongruent features of category members. Journal of Experimental Psychology - Learning Memory and Cognition, 30(5), 1065-1081.

Lawal, B. (2003). Categorical Data Analysis with SAS and SPSS Applications. Mahwah, NJ: Lawrence Erlbaum Associates. 
Leighton, J. P., \& Sternberg, R. J. (2004). The Nature of Reasoning. Cambridge, England: Cambridge University Press.

Margolis, E., \& Laurence, S. (1999). Concepts: Core Readings. Cambridge, MA: MIT Press.

Novak, J. D. (1990). Concept maps and Vee diagrams - 2 metacognitive tools to facilitate meaningful learning. Instructional Science, 19(1), 29-52.

Perkins, D. N., \& Simmons, R. (1988). Patterns of misunderstanding-An integrative model for science, math, and programming. Review of Educational Research, 58(3), 303-326.

Preece, P. F. W. (1976). Mapping cognitive structure: A comparison of methods. Journal of Educational Psychology, 68, 1-8.

Sanger, M. J. (2000). Using particulate drawings to determine and improve students' conceptions of pure substances and mixtures. Journal of Chemical Education, 77(6), 762-766.

Schummer, J. (1998). The chemical core of chemistry I: A conceptual approach. HYLE - International Journal for Philosophy of Chemistry, 42, 129-162. Retrieved August 25, 2005, from http://www.hyle.org/journal/issues/4/schumm.htm

Shafto, P., \& Coley, J. D. (2003). Development of categorization and reasoning in the natural world: Novices to experts, naive similarity to ecological knowledge. Journal of Experimental Psychology - Learning Memory and Cognition, 29(4), 641-649.

Smith, C., Carey, S., \& Wiser, M. (1985). On differentiation: A case study of the development of size, weight, and density. Cognition, 21(3), 177-237.

Symington, D., Boundy, K., Radford, T., \& Walton. J. (1981). Children's drawings of natural phenomena. Research in Science Education, 11, 44-51.

Taber, K. (2002). Chemical Misconceptions - Prevention, Diagnosis and Cure. Volume I: Theoretical Background. London: Royal Society of Chemistry.

Talanquer, V. (2006). Common sense chemistry: A model for understanding students alternative conceptions. Journal of Chemical Education, 83(5), 811.

Tanaka, J. W., \& Taylor, M. (1991). Object categories and expertise-Is the basic level in the eye of the beholder. Cognitive Psychology, 23(3), 457-482.

Todd, P. M., \& Gigerenzer, G. (2000). Précis of simple heuristics that make us smart. Behavioral and Brain Sciences, 23(5), 727-780.

Tsai, C. C., \& Huang, C. M. (2002). Exploring students' cognitive structures in learning science: A review of relevant methods. Journal of Biological Education, 36(4), 163-169.

Tversky, A., \& Kahneman, D. (1982). Judgment under uncertainty: Heuristics and biases. In D. Kahneman, P. Slovic, \& A. Tversky (editors), Judgment under Uncertainty: Heuristics and Biases. New York: Cambridge University Press.

Viennot, L. (2001). Reasoning in Physics: The Part of Common Sense. Dordrecht, The Netherlands: Kluwer Academic.

Vosniadou, S. (1994). Conceptual change. Learning and Instruction, 4, 45-69.

Vosniadou, S. (2002). On the nature of naive physics. In: M. Limón \& L. Mason (editors), Reconsidering Conceptual Change: Issues in Theory and Practice. Dordrecht, The Netherlands: Kluwer Academic.

Wandersee, J. H., Mintzes, J. J., \& Novak, J. D. (1994). Research on alternative conceptions in science. In D. Gabel \& National Science Teachers Association (editors), Handbook of Research on science teaching and learning. New York: Macmillan.

Wellman, H. M., \& Gelman, S. A. (1998). Knowledge acquisition in foundational domains. In: D. Kuhn \& R. S. Siegler (editors), Handbook of Child Psychology. Volume 2: Cognition, Perception, and Language. New York: John Wiley \& Sons.

White, R., \& Gunstone, R. (1992). Probing Understanding. London: The Falmer Press. 